Cell Research (2003); 13(2):69-81

http://www.cell-research.com

\title{
Function and regulation of Aurora/Ipl1p kinase family in cell division
}

\author{
Yu Wen KE ${ }^{1}$, Zhen DOU ${ }^{1}$, Jie ZHANG ${ }^{1}$, Xue BiAo YAO ${ }^{1,2, *}$ \\ ${ }^{1}$ Laboratory for Cell Dynamics, School of Life Sciences, University of Science and Technology of China, Hefei \\ 230027, China \\ ${ }^{2}$ Department of Molecular and Cell Biology, University of California, Berkeley, CA 94720, USA
}

\begin{abstract}
During mitosis, the parent cell distributes its genetic materials equally into two daughter cells through chromosome segregation, a complex movements orchestrated by mitotic kinases and its effector proteins. Faithful chromosome segregation and cytokinesis ensure that each daughter cell receives a full copy of genetic materials of parent cell. Defects in these processes can lead to aneuploidy or polyploidy. Aurora/Ipl1p family, a class of conserved serine/threonine kinases, plays key roles in chromosome segregation and cytokinesis. This article highlights the function and regulation of Aurora/Ipl1p family in mitosis and provides potential links between aberrant regulation of Aurora/Ipl1p kinases and pathogenesis of human cancer.
\end{abstract}

Key words: Aurora (Ipl1p), mitosis, and cancer.

\section{INTRODUCTION}

Cell is a fundamental unit of life that is relayed via mitosis. The essence of mitosis is to segregate parental genomes encoded in sister chromatides into two daughter cells, such that each of them inherits one complete copy of genome. In addition, each daughter cell must receive one centrosome and the equal complements of cytoplasm and organelles. Mitosis is often catagorized into five distinct stages (determined by the configuration of mitotic spindle and chromosomes; Fig 1): prophase, prometaphase, metaphase, anaphase and telophase. Cytokinesis, the process of daughter cell separation, occurs at the end of mitosis, and regulated by the machinery governing mitotic progression and exit. A healthy mitosis depends on two fully functional centrosomes, spindle microtubule and kinetochore, a multi-protein complex assembled onto centromeric DNA of chromosome. Although the exact temporal and spatial coordination of mitosis and cytokinesis differ

* Correspondence: Prof. Xue Biao YAO

Tel: 0086-551-3607141 Fax: 0086-551-3607141

E-mail: yaoxb@ustc.edu.cn among species, the regulatory machinery is conserved from yeast to human. One of most conserved regulators is serine/theronine protein kinase superfamily that alters the function of its effectors via protein phosphorylation. Entry into mitosis is driven by protein kinases while initiation of exit from mitosis is tied to regulated proteolysis. These are interrelated processes in which the proteolysis is regulated by phosphorylation while several kinases are in turn shut off by regulated proteolysis for the mitotic exit.

There are several protein kinase families that play distinct roles in different stages of mitosis[1]. The mitotic master kinase is cyclin B-dependent kinase (Cdk1), the founding member of the Cdk family of cell cycle regulators. CDK-cyclin complex can catalyze protein phosphorylation on an array of protein effectors bearing diversified biological function, ranging from DNA replication to mitosis. The entry into mitosis is governed by Cdc2-cyclin $\mathrm{B}$. The second family is polo-like kinases (PLKs), they involve in centrosome maturation, activation and inactivation of Cdk1, and cytokinesis. The third family is named NIMA (never in mitosis A) family, implicated 


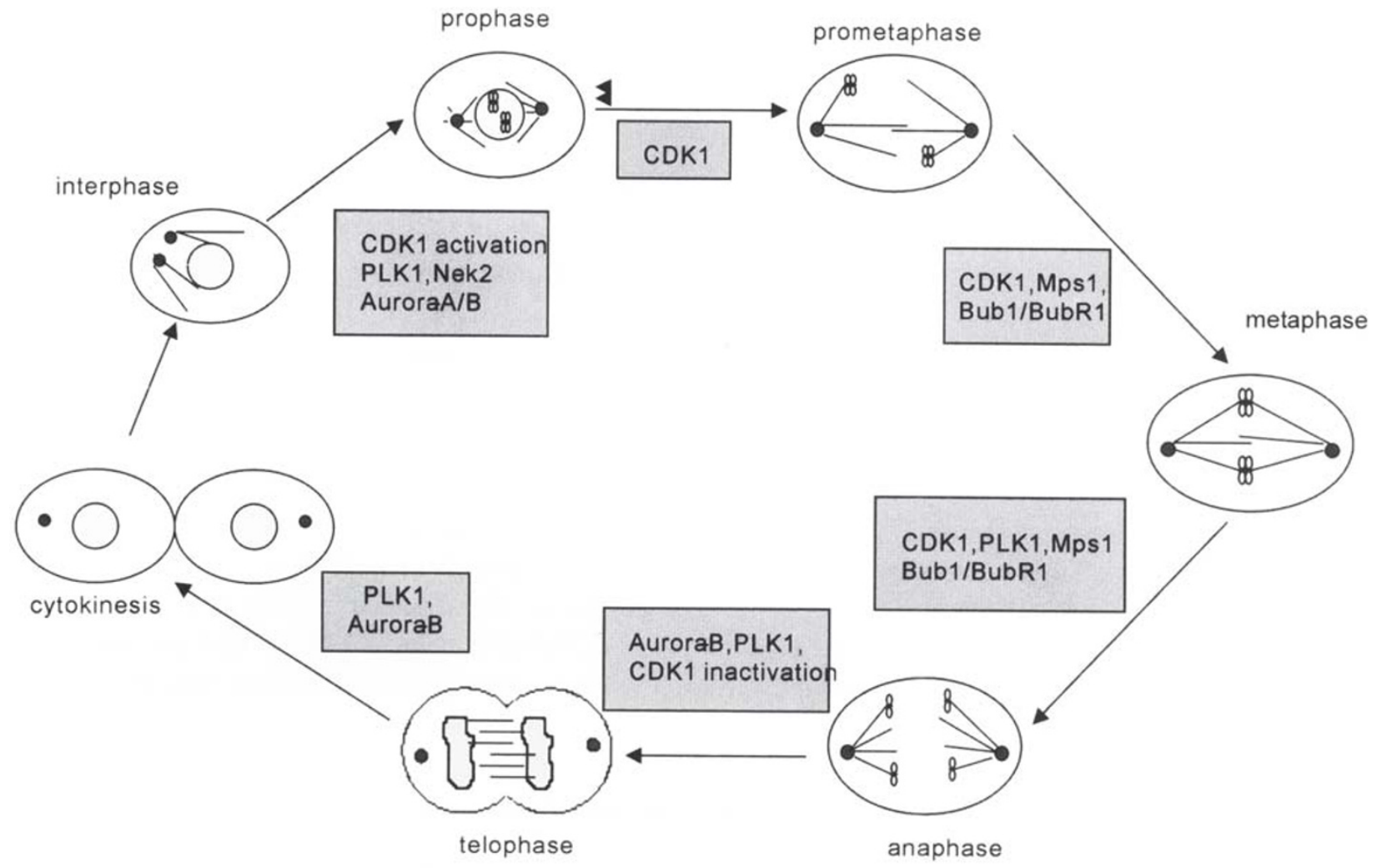

Fig 1. Cell cycle and kinase signaling cascades

in centrosomes duplication and separation. The fourth family includes Bub1 (budding uninhibited by benomyl) and Mps1 (mono-polar spindle)[2] kinases that play active roles in mitotic checkpoint signaling. The fifth family is called Aurora/IpI1p kinases, active in regulating chromosome segregation and cytokinesis. This review will focus on the function and regulation of Aurora/IpI1p protein kinase family. Interesting readers for other mitotic kinases are referred to related review articles[e.g., Ref. 1].

\section{Aurora/Ipl1-related kinases in different organisms}

The founding members of the Aurora kinase family are IpI1p from budding yeast S. cerevisiae, based on a pioneering genetic screen for mutations that lead to increase in chromosome mis-segregation [3], and Aurora from Drosophila melanogaster[4]. While IpI1p is the only Aurora kinase in yeast, there are two Aurora kinases in Drosophila melanogaster, and C. elegans[5, 6], and at least three members in human[7]. All Aurora/IpI1p kinases share similar catalytic domains located in the carboxyl end with great diversity in their amino-terminals. In mammals, three Aurora kinases are found: AuroraA, Aurora-B, and Aurora-C[7]. Besides the difference in the primary structure of these three kinases, their subcellular localization and functional regulation are distinctly different (Tab 1). Since this review is written mainly from the perspective of Aurora kinases in human, for the sake of clarity, we adapted the nomenclature proposed by Bischoff and Plowman[7] and substantiated by Nigg[1].

The three human Aurora kinases range from 309 to 403 amino acids and share $67-76 \%$ amino acid sequence identity in their catalytic domains with little similarity in their $\mathrm{N}$-terminus that provide molecular basis for specific but diversified interactions with different effector proteins. These Aurora-effector protein interactions may account for their distinct subcellular localization on mitotic spindle. The Aurora family members share highly conserved sequence in the catalytic domain of the kinase across different organisms. The overall sequence identities between human and rodent proteins are: Aurora-A 
Tab 1. Substrates and partners for Aurora kinases

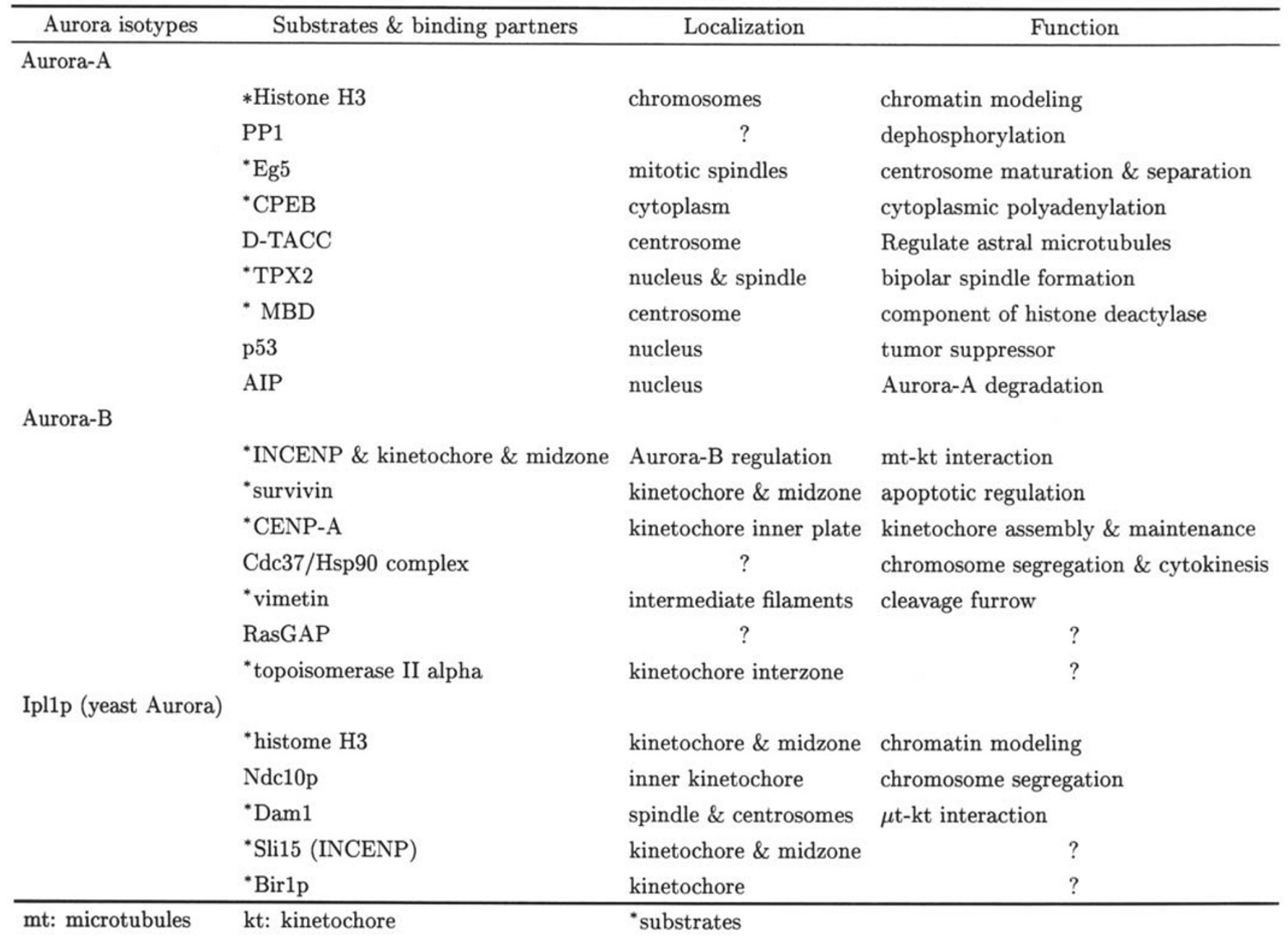

(82\%), Aurora-B (84\%) and Aurora-C (78\%), respectively [Fig 2 ; ref. 7].

The high conservation of Aurora kinases among different organisms implies a similar regulatory mechanisms underlying Aurora function in cell division cycle. Both Aurora-A and Aurora-B are preferentially expressed in proliferating cells and overexpressed in tumor cells. While the enzymatic activities of these two kinases bearing distinct temporal regulation in mitosis, the two kinases also have different subcellular distribution profile[8]. AuroraA is chiefly associated with centrosome in interphase and mitotic cells while Aurora-B is mainly located at kinetochore of mitotic cells and to the midbody of cytokinetic cells. Aurora-C is located to centrosomes only in telophase cells. Overall distribution of $\mathrm{Au}-$ rora kinases superimposes to that of PLKs, raising the possibility of cross-signaling between the two kinase families.

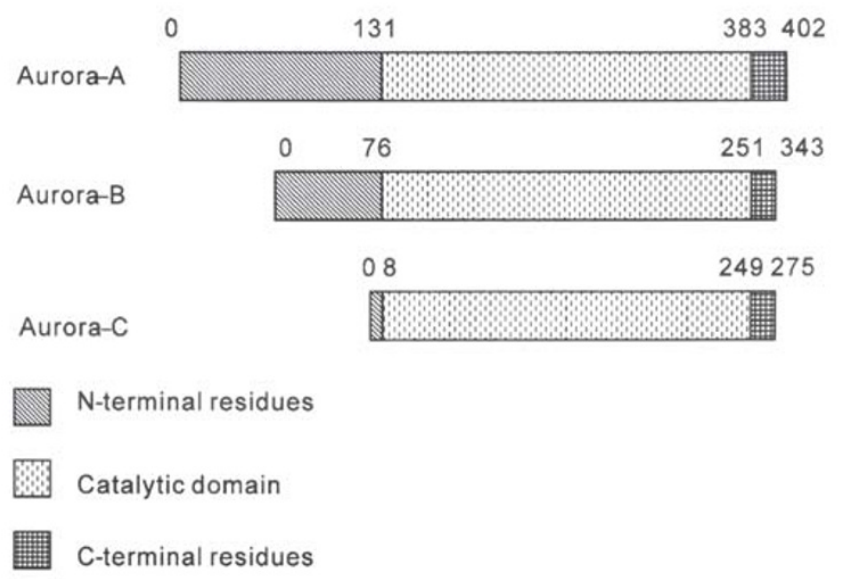

Fig 2. Structure of Aurora kinases 


\section{Aurora-A}

Aurora-A expresses abundantly in cells with high mitotic or meiotic index, such as thymus, embryo and over-expresses in several cancer cell lines. The protein accumulation of Aurora-A varies across the cell cycle, low in G1/S, peaks in G2/M, and then declines after mitosis due to regulated proteolysis $[9,10]$.

Aurora-A involves in centrosome maturation and spindle assembly

When cell enters mitosis, centrosomes mature with the accumulation of $\gamma$-tubulin and PCM materials. Genetic studies from C. elegan suggest that Aurora-A (AIR-1) is not required for the onset of centrosomes separation, but it is essential for the maintenance of centrosomes separation. When Aurora-A was eliminated by RNAi, centrosomal $\gamma$ tubulin fluorescence intensity was only $40 \%$ compared to that of wild-type, suggesting a defect in centrosomal maturation[11]. Furthermore, Aurora$\mathrm{A}$ is required for the accumulation of centrosomal gtubulin and other two PCM components as embryo enters mitosis. In Drosophila, mutations in Aurora lead to unseparated centrosome and monopolar spindle[4]. In Xenopus, Eg2 (the homolog of AuroraA) situates around PCM during interphase, but relocates on spindle microtubule in anaphase[12]. It has been demonstrated that Eg2 binds to spindle microtubule in vitro, and it is required for bipolar spindle formation.

\section{Interaction between Aurora-A and PP1 phos- phatase}

Several lines of evidence indicate that Ipl1p kinase interacts with Glc7 (the catalytic subunit of protein phosphatase 1) and both involve in chromosome separation in budding yeast[13, 14]. In addition, it was reported that Glc8 (the homologue of inhibitor 2) regulates budding yeast PP1 activity [15]. In mammalian cells, it has been shown that Aurora-A interacts with PP1 through protein phosphorylation and in turn PP1 inactivates Aurora-A by dephosphorylation of the kinase[16]. Mutation analysis indicated that disruption of Aurora-A-PP1 induced hyper-phosphorylation of Aurora-A in mitosis, which led to a great reduction of the kinase activity. These results suggest that: 1) single or mul- tiple inhibitory phophorylation sites as well as activation sites exist on Aurora-A; 2) these sites are phosphorylated by unknown inhibitory kinases and are dephosphorylated by PP1 at the onset of mitosis, leading to Aurora-A activation. Recent studies from Xenopous egg extracts have identified that Thr295 and Ser349 are phosphorylated and elimination of Thr295 effects loss of kinase activity while mutation of Ser349 completely block activation of Aurora$\mathrm{A}[17]$. While these results help us to understand the function of human Aurora-A, it would be of great interests to identify precise amino acids on AuroraA subjected to mitotic phosphorylation and consolidate these modifications to spatial regulation of $\mathrm{Au}$ rora-A function in mitosis.

\section{The cell cycle-regulated Aurora degradation}

The Aurora-A activity is down-regulated by mitotic degradation through APC/C-dependent proteolysis[18, 19]. There are two forms of APC/C activated sequentially by the association with two distinct WD40 repeat proteins known as Cdc20 and $\mathrm{Cdh} 1$, respectively. $\mathrm{APC} / \mathrm{C}^{\mathrm{Cdc} 20}$ is active at the metaphase-anaphase transition while $\mathrm{APC} / \mathrm{C}^{C d h 1}$ activity is turned on later in mitosis and remains high through G1 interphase. Interestingly, mitotic kinases regulate the two forms of $\mathrm{APC} / \mathrm{C}$ in opposite fashion that provides temporal order of $\mathrm{APC} / \mathrm{C}$ activity. While phosphorylation of APC/C core subunit and perhaps Cdc20 is required for activation of $\mathrm{APC} / \mathrm{C}^{\mathrm{Cdc} 20}$, phosphorylation of $\mathrm{Cdh} 1$ prevents the activation of $\mathrm{APC} / \mathrm{C}^{\mathrm{Cdh} 1}[20]$.

Recent biochemical and genetic studies shed lights on the degradation mechanism of Aurora-A [21-23]. Several lines of evidence indicated that the degradation is dependent on $\mathrm{APC} / \mathrm{C}^{C d h 1}$ but not $\mathrm{APC} / \mathrm{C}^{C d c 20}$. The C-terminal D box in Aurora-A is required for Cdh1-induced destruction while the $\mathrm{N}$ terminal KEN box is not. Destruction also requires a short stretch named DAD (D-Box-activating domain) located to the N-terminus, whose deletion arrests the APC/ $\mathrm{C}^{C d h 1}$-mediated degradation.

Recently, Kiat et al. (2002) had identified a novel negative regulator of Aurora-A named AIP (AuroraA interacting protein) based on a dosage suppressor screen in yeast[24]. AIP is a ubiquitously expressed nuclear protein that interacts specifically with hu- 
man Aurora-A in vivo. Interestingly, ectopic expression of AIP with Aurora-A in NIH 3T3 and COS cells results in the down-regulation of ectopically expressed Aurora-A protein levels, and this downregulation is mediated by a non-26S proteasomemediated degradation of Aurora-A. A non-interacting deletion mutant of AIP does not down-regulate Aurora-A protein, suggesting that the interaction is important for the protein degradation. It would be of great interest to test whether association of AIP with Aurora-A induces conformational changes of Aurora-A that facilitate the proteasome-substrate recognition and subsequent proteolysis. Since AIP is also expressed in a large collection of cancer cell lines, it is likely that additional negative regulators account for mechanisms underlying Aurora-A overexpression.

Substrates and partners for Auroras (summarized in Tab 1)

Using cell-free extracts prepared from Xenopus egg, Giet et al. (1999) demonstrated that Aurora-A (Eg2) interacts with and phosphorylates Eg5, an important motor protein for centrosome maturation and separation[25]. It remains to be established the physiological function of such phosphorylation on bipolar spindle assembly as well as maintenance. Another important substrate of Aurora-A is CPEB (cytoplasmic polyadenylation element binding factor). Aurora-A phosphorylates CPEB at Ser174. This phosphorylation is very important for C-mos mRNA polyadenylation and its translation subsequently triggers egg maturation[26, 27].

Experimentation from Drosophila shows that activation of the Aurora-A kinase at the onset of mitosis is required for the actin-dependent asymmetric localization of $\mathrm{Numb}$ [28], suggesting that Aurora-A regulates both actin- and microtubuledependent processes in mitotic cells. In addition, a portion of the Drosophila Aurora-A forms a complex with D-TACC, a microtubule-binding protein[29]. Mutational analysis of Aurora-A revealed that the kinase is required for targeting D-TACC to centrosomes and regulating astral microtubules.

Recently, Kufer et al. (2002) revealed that Nterminal domain of TPX2, a prominent component of the spindle apparatus, can directly interact with the C-terminal catalytic domain of Aurora-A. In addition, these authors showed that TPX2 is a substrate for Aurora-A. Furthermore, they demonstrated that TPX2 is required for the targeting of Aurora-A to spindle microtubules but not for its localization to centrosomes[30]. Interestingly, Gruss et al. (2002) show that spindle formation requires the function of TPX2 to generate a stable bipolar spindle with overlapping antiparallel microtubule arrays[31]. However, it remains to be established the physiological relevance of the TPX2 phosphorylation in bipolar spindle formation during mitosis and whether Aurora-A mediated phosphorylation alters the properties of TPX2 $\times$ microtubule interaction.

MBD3, a component of the histone deacetylase $\mathrm{NuRD}$ complex, was also found to interact with $\mathrm{Au}$ rora-A and colocalized with it on the centrosomes [32]. In addition, MBD3 is a substrate of Aurora-A. Interestingly, HDAC1 is distributed at the centrosomes in a manner similar to MBD3. These results suggest the highly dynamic nature of the temporal and spatial distributions of the NuRD complex in $\mathrm{M}$ phase, probably through an interaction with mitotic kinases such as Aurora-A.

Using a trans-activation-defective p53 derivative as bait, Chen et al. isolated Aurora-A (STK15) as a p53-binding partner[33]. The p53- Aurora-A interaction was confirmed further by co-immunoprecipitation and GST pull-down studies. In a subsequent co-transfection experiment, these authors show that p53 suppresses Aurora-A-induced centrosome amplification and cellular transformation in a transactivation-independent manner. The suppression of Aurora-A oncogenic activity by p53 might be explained in part by the finding that p53 inhibites Aurora-A kinase activity via direct interaction with the catalytic domain of the kinase[33]. This raises a very interesting question as whether mutant p53 fails to interact with Aurora-A, which effects an elevation of Aurora kinase activity in certain cancer cells.

Taken together, these studies demonstrate that Aurora-A play a significant role in centrosome maturation and spindle assembly by forming functional complexes with other regulatory proteins. Perturbation of the aforementioned protein-protein interactions alters the faith of cell division.

\section{Aurora-B}


The protein expression level of Aurora-B is high during anaphase in tissues with a high mitotic index such as thymus and fetal liver. In mitotic cells, Aurora-B is localized to the kinetochore of prometaphase chromosome and to the spindle midzone of anaphase cells. Mutation of Aurora-B can lead to defects in chromosome separation and cytokinesis, indicating its essential role in a faithful cell division.

\section{Chromosomal passenger proteins}

Chromosomal passengers are proteins that move from centromeres to the spindle midzone during mitosis. The success of mitosis depends on a large degree on the interaction between chromosome and spindle microtubules. Chromosomal passenger can coordinate their functions. These proteins associate with chromosomes along their long arm during prophase, becoming concentrated at the inner centromere in metaphase. Upon the transition from metaphase to anaphase, the passenger proteins transfer to the central region of the mitotic spindle and to the cell cortex in the region where the contractile ring will form. Based on the distribution profile, it is likely that these proteins may involve in biogenesis of functional centromere during early mitosis, and then perform distinct but essential functions during anaphase and telophase, perhaps modulating the activities of microtubule-binding proteins [34].

INCENP (inner centromere protein), Aurora-B, and survivin are three known chromosomal passengers that often functions as a complex[35-36], mutations altering the complex will lead to defects in chromosome separation and cytokinesis. INCENP and Aurora B have identical localization patterns during mitosis and directly bind each other in vitro. Recently, Bishop and Schumacher show that the C. elegans Aurora-B (AIR-2) specifically phosphorylates the C. elegans INCENP (ICP-1) at two adjacent serines within the carboxyl terminus[37]. Furthermore, the full length and a carboxyl-terminal fragment of INCENP stimulated Aurora-B kinase activity judged by the incorporation of ${ }^{32} \mathrm{P}$ into histone H3. This increase in Aurora-B activity required Aurora-B-mediated phosporylation on ICP1 as mutation of both phosphorylatable serines on the INCENP abolished its stimulatory effects on
Aurora-B kinase activity. Thus, INCENP is directly phosphorylated by Aurora-B and functions in a positive feedback loop that up-regulates Aurora-B kinase activity[37-38]. Similar inter-relationship was also found between survivin and Aurora-B both in vitro and in vivo[39]. Interestingly, Chen et al. (2003) demonstrated that depletion of survivin protein by antisense oligonucleotide treatment suppressed the phosphorylation of histone H3 and caused mislocalization of Aurora-B, verifying synergy in Aurora-B activity when the binary complex forms [39]. The current working model for this protein complex is illustrated in Fig 3 . It would be interesting to see whether synergy or antagonism in Aurora-B activity exists in the ternary complex formed by Aurora-B, survivin and INCENP.

Gigoux et al. have recently identified Ras GTPase-activating protein, as a binding partner of Aurora-B[40]. Interestingly, these authors further show that RasGAP forms a ternary complex with Aurora-B and survivin. Since survivin situates on the crossroad of cycling and apoptosis, it would be of great interest to distinguish the functional specificity of the two aforementioned ternary complexes in deciding to continue cycling or exit from the cell cycle.

Functional studies of yeast Aurora-B-INCENP protein complex

Aurora-B-INCENP protein complex is evolutionally conserved from yeast to human[7]. Recent studies on yeast Aurora-B-INCENP (IpI1pSli15) were fueled by the importance of human homologues in cell division. Genetic studies revealed that budding yeast INCENP (Sli15) is essential for facilitating Aurora (Ipl1p) function in chromosome segregation[41]. Kang et al. revealed that the binary complex of INCENP-Aurora associates with microtubule binding protein Dam1, a substrate of Aurora, indicating that the complex may play a pivotal role in the regulation of chromosome-spindle microtubule interaction[42, 43]. For the proper segregation of sister chromatids before cell division, each sister kinetochore must attach to microtubules emanating from opposite spindle poles, so called bi-orientation. The chromosome bi-orientation is crucial for proper chromosome segregation, but is still poorly understood. Tanaka et al. recent showed that Au- 
rora-INCENP protein complex plays an important role in chromosome bi-orientation[44]. In Aurora or INCENP mutant cells, frequency of the attachment of sister kinetochores to spindle microtubule emanating from same microtubules is extremely high. Interestingly, the spindle checkpoint is not apparently linked to the machinery as mutation and elimination of spindle checkpoint proteins such as Mad2 have little effect on the spindle bi-orientation in yeast. Thus, it would be of great interest to illustrate the molecular interaction of Aurora-INCENP protein complex at the kinetochore and consolidate these interactions into the regulation of kinetochore biorientation.

Aurora-B is required for chromosome separation and cytokinesis

After DNA replication, chromatins condense and assemble onto histone to form chromosome to facilitate faithful transmission of genome into two daughter cells during mitosis. Phosphorylation of histone $\mathrm{H} 3$ at serine 10 occurs during mitosis in diverse eu-

\section{A. Action of the chromosomal passenger complex on chromatin}

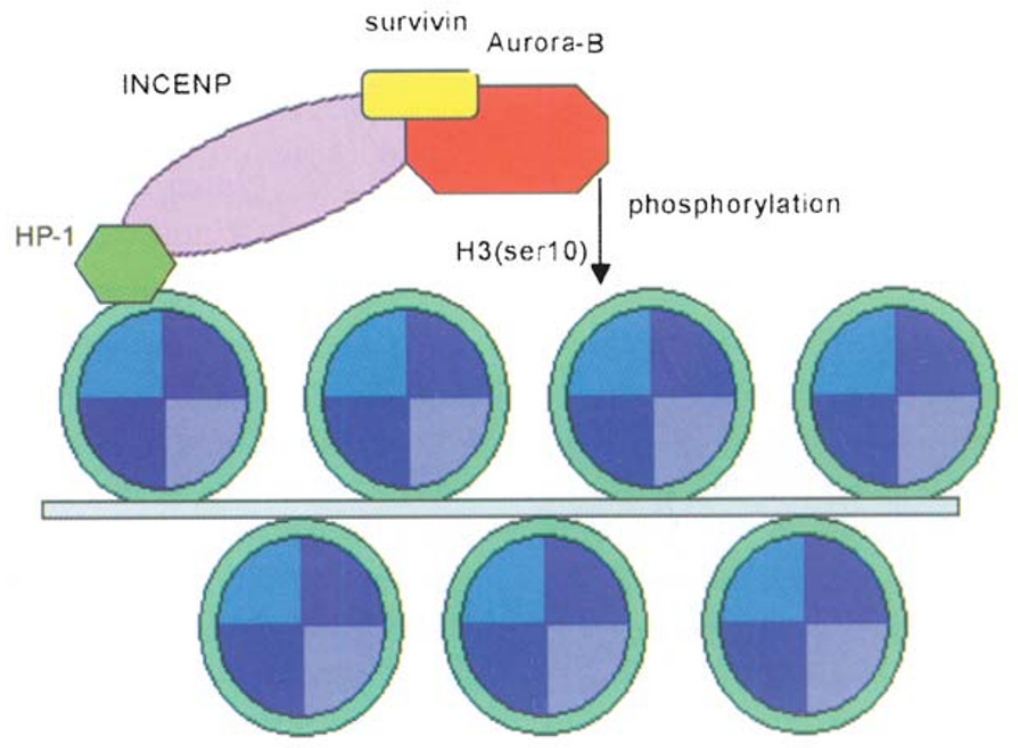

B. Action of the chromosomal passenger complex on the central spindle

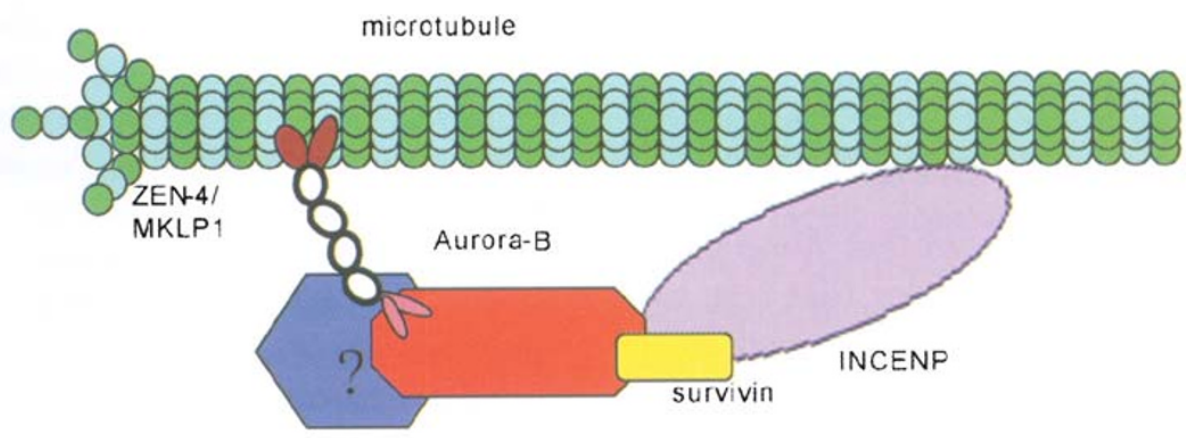

Fig 3. Role of the chromosomal passenger complex on chromosomes and the central spindle[32]. A. INCENP targe Aurora-B to histone H3 through associating with heterocromatin protein HP-1, coordinating survivin. B. During the transition from anaphase to telophase, this INCENP-survivin-AuroraB complex is required for tareting motor protein ZEN-4/MKLP 1 to spindle midzone

karyotes and correlates closely with mitotic and meiotic chromosome condensation. Failure in phosphorylated $\mathrm{H} 3$ showed abnormal chromosome segregation, resulting in extensive chromosome loss during mitosis, indicating that $\mathrm{H} 3$ serine 10 phosphorylation is causally linked to chromosome con- 
densation and segregation in vivo and is required for proper chromosome dynamics $[45,46]$. In fact, this modification is also conserved in budding yeast and C. elegans. Aurora and PP1 are the respective upstream kinase and phosphatase which regulate the modification of histone H3[47]. Crosio et al. show that both Aurora-A and Aurora-B proteins physically interact with the H3 tail and efficiently phosphorylate Ser10 both in vitro and in vivo[48]. In addition, Aurora-B also can phosphorylate H3 on the site Ser28[49], it remains to be established the physiological function of phosphorylation of Ser28 in mitosis.

Human CENP-A, a variant of histone 3, contains a phosphorylation motif that conserved between histone 3 and CENP-A (e.g. Ser10). In fact, Lertlin and colleagues confirmed that Aurora-B phosphorylates CENP-A and the phosphorylation site was similar to histone H3. Expression of dominantly negative non-phosphorylatable mutant CENP-A in human cells results in a delay at the terminal stage of cytokinesis and an error in subcellular targeting Aurora-B, INCENP and PP1[50, 51]. These data support a model where CENP-A phosphorylation is involved in regulating the aforementioned protein targeting within the cell. In addition, these studies point out the importance of specific site phosphorylation on histone dynamics underlyinging kinetochore assembly in prometaphase and disassembly at the end of anaphase B for DNA de-condensation. In any event, it now possible to monitor kinetochore dynamics using fluorescence reporter such GFPCENP-B to visualize the real-time cell division to ascertain the precise regulatory mechanism.

Recently, Lange et al. showed that Aurora-B interacts with CDC37/Hsp90 complex that is in turn required it for maintaining the activity and stability of Aurora-B[52]. Cdc37/Hsp90 complex modulates the function of Aurora B and that most of the phenotypes derived from the loss of Cdc37 function can be explained by the inactivation of this kinase. These observations substantiate the role of Cdc37 as an upstream regulatory element of key cell cycle kinases [52].

In addition, Goto et al. recently revealed that Aurora-B controls the cleavage furrow-specific vimentin phosphorylation in the cytokinesis[53].
These authors carried out detailed mutational analyses and concluded that four Aurora-B-mediated phosphorylation sites are important for the bridge formation. Expression of non-phosphorylatable vimentin mutants display phenotype of an unusual long bridge-like intermediate filament structure between un-separated two daughter cells[53], indicating that cleavage furrow-specific vimentin phosphorylation important for cytokinetic process. It would be very informative to ascertain if alternation of the aforementioned phosphorylation sites slow down the disaasembly kinetics of vimentin filaments. In any event, it becomes apparent that Aurora-B targets to the right place at right time to perform several distinctly different function during mitosis.

Aurora-B kinase activation is required for microtubule-kinetochore interaction

Functional role of Aurora-B in association of spindle microtubules with kinetochore was carefully examined by antibody perturbation experiment in cultured mammalian cells[54]. Injection of AuroraB kinase inhibitory antibody caused failure in chromosome congression, consistent with a conserved role for Aurora B in bipolar attachment of chromosomes. In addition, inhibition of Aurora-B in cultured cells and in cycling Xenopus egg extracts caused escape from the spindle checkpoint arrest induced by microtubule drugs, implicating Aurora$\mathrm{B}$ as a critical coordinator relating changes in microtubule dynamics in mitosis, chromosome dynamics, and signaling of the spindle checkpoint. To pursue the mechanisms underlying these defects, MurataHori and Wang have examined chromosomal movement, spindle organization, and microtubule motor distribution in NRK cells expressing a kinaseinactive, dominant-negative mutant of Aurora B (DN-Aurora)[55]. In cells overexpressing GFPcoupled DN-Aurora, centromeres displayed a synchronized and unidirectional movement as opposed to the independent, bidirectional oscillation in control cells. Interestingly, most kinetochores became physically separated from spindle microtubules, which are seen as a striking bundle between the spindle poles. These defects were associated with eliminations of microtubule motor proteins dynein and CENP-E from kinetochores, suggesting that 
Aurora B regulates the association of motor proteins with kinetochores during prometaphase. Since CENP-E is phosphorylated by mitotic kinase[56] and the phosphorylation eliminates the binding of CENP$\mathrm{E}$ to microtubules, elimination of Aurora-B may affect a phenotype of loss of CENP-E in which mitotic spindle is deformed and fragile[57]. It would be of great interest to test whether CENP-E is a substrate for Aurora-B and elucidate the physiological regulation of CENP-E by Aurora during mitosis. Given the spatial and temporal co-distribution profiles of the two proteins [58, 7], and phosphorylation profile of CENP-E during cell cycle [Yao et al, unpublished observation], it becomes even more plausible to establish the inter-relationship between Aurora kinase and motor and consolidate their interactions into chromosome dynamics during prometaphase and potential role of CENP-E in anaphase chromosome movements and spindle elongation[56-58].

Using genetic tractable organism budding yeast coupled to the advancement in mass spectrometric analysis, Chesseman et al. have purified 28 kinetochore proteins from yeast protein extracts as yeast Aurora (Ipl1p) interacting proteins, including five previously uncharacterized kinetochore proteins. Using mass spectrometry, these authors further identified 7 substrates from these 28 proteins. Mapping these phosphorylation sites targeted directly by yeast Aurora has generated a consensus phosphorylation sequence motif for an Aurora kinase. Interestingly, their analyses show that an essential microtubule binding protein Dam1p is a key Ipl1p target for regulating kinetochore-microtubule attachments in vivo [43]. Additional work along this line will help to clarify the molecular regulation of yeast kinetochore protein complex by Aurora kinase during mitosis. Given the conservation of essential kinetochore proteins including Aurora kinase from yeast to mammals, it would be interesting to see whether the aforementioned yeast kinetochore regulatory networks are conserved in human despite the distinct ultrastructural architectures between the organisms.

\section{Aurora-C}

Among three human Aurora kinases, there are few functional studies about Aurora-C. Northern blot analysis revealed that expression of Aurora-C is limited to testis. The expression levels of Aurora-C in several cancer cell lines increased several-fold compared with normal fibroblasts. In HeLa cells, the Aurora-C protein level is low in G1/S, accumulates during $\mathrm{G} 2 / \mathrm{M}$, and reduces after mitosis, similar to these of Aurora-A and Aurora-B. Immunofluorescence studies showed that Aurora-C is localized to centrosomes from anaphase to cytokinesis[59]. These results suggest that Aurora-C may play a role in centrosome function during the later stages of mitosis.

\section{Aurora and cancer}

The biology of mitosis has intrinsic appeal for cell biologists as well as clinical physicians who are keen on the pathogenesis and treatment of cancer. Most aggressive human cancers are featured by an inherent instability of their genomes associated with cell division, a phenotype named genomic instability[60]. The most common type of instability is seen cytologically as chromosomal instability that is present in all classes of solid tumor[61]. In fact, chromosomal instability constitutes the hallmark of cancer, which is evident by chromosome loss, translocation and aberrant centrosome duplication, indicating an underlying deregulation in chromosome segregation, centrosome duplication and segregation[62]. Mutation in chromosomal stability genes (e.g., Bub1) increases the probability that whole chromosomes or large fractions of chromosomes are gained or lost during cell division. The consequence of chromosome instability is an imbalance in the number of chromosomes per cell (aneuploidy) and an enhanced rate of loss of heterozygosity. While chromosomal aberrations are particular common in advanced, highly invasive cancers, a similar situation is true for centrosomal abnormalities. Genetic analyses show that mutations in the genes that encode p53 or other key G2/M-phase checkpoint proteins (e.g., Bub1) occur in cancer cells and result in genomic instability[63]. Several other genes that are involved in mitotic regulation have been shown to be overexpressed in tumors (e.g., Aurora-A, B, C, and survivin).

Tab 2 summarizes gene mapping, amino acid length of Aurora kinases, and their aberrant expression in various cancer cell lines. Several studies indicate that Aurora-A has a close link to cancer pathogenesis[64-68]. The Aurora-A gene was mapped to chromosome 20q13.2-13.3, a region often amplified in human cancers[69]. In addition, it has been re- 
ported that the level of Aurora-A mRNA is high in colon cancer[64, 65], as well as in cell lines derived from breast, ovarian, colon, prostate, neuroblastoma and cervical cancers[66]. Ectopic expression of Aurora-A in mouse NIH3T3 cells display the phenotype of abnormal centrosomes replication and cell transformation. Moreover, it has been shown that over-expression of Aurora-A in diploid human breast epithelial cells causes aberrant centrosome replication, as well as induction of aneuploidy, indicating that there are intrinsic links between aberrant regulation of Aurora-A activity and pathogeneses of aneuploidy and chromosomal instability[70, 71]. Recently, Meraldi et al. show that over-expression of Aurora-A gave rise to centrosome amplifica- tion and polyploid[72]. Interestingly, both phenotypes was enhanced in a p53-/- background, indicating that p53-dependent checkpoint may function at eliminating cells underwent aberrant cell division [73].

Why a single mitotic protein kinase such as Aurora-A is able to transform cells and behaved as oncogene? One possible explanation is that over-expression of Aurora-A exceed the threshold of functional mitotic checkpoint surveillance, which leads to abnormalities in cell division and subsequent loss of tumor suppressor genes or the amplification of oncogenes, which subsequently effects a transformation of normal cells into tumor cells.

Aurora-B gene is mapped to $17 \mathrm{p} 13$, a region of-

Tab 2. Aurora over-expression in cancer cells

\begin{tabular}{llcl}
\hline Name & Gene mapping & AA length & Overexpressing in \\
\hline Aurora-A & $29 \mathrm{q} 13.2-13.3$ & 403 & $\begin{array}{l}\text { Breast, orarian, colon, prostate, } \\
\text { neuro blastoma, and cervical cell lines }\end{array}$ \\
Aurora-B & $17 \mathrm{p} 13$ & 344 & Colorectal cell lines \\
Aurora-C & $19 \mathrm{q} 13.3$ & 309 & HepG2, HuH7, MDA-MB-453, Hela \\
\hline
\end{tabular}

ten deleted in various human cancers. Overexpression of Aurora-B was found in colorectal tumors that display polyploidy. The level of Aurora-B protein becomes high in several cancer cell lines, and its binding protein INCENP level also rises greatly in some colorectal cell lines[74], consistent with the notion that Aurora-B activity is elevated in tumor cells as INCENP is a stimulatory factor for Aurora-B kinase.

Aurora-C was mapped to $19 q 13.4$, a region highly susceptible to translocation, deletion and amplification, which have been reported in various cancers. Interestingly, Aurora-C expression is very restrictive to certain cancer cells. So far, it remains uncovered the relationship between tumorgenesis and Aurora-C expression.

\section{Future directions}

Although great progress has been made over the last 2 years in characterizing the mechanisms and participants in Aurora kinase signaling networks, the challenge ahead is to define the precise function and the physiological regulation of the implicated proteins as well as to identify new players. By analyzing both cell biology of Aurora kinases mitotic regulation and phenotypic changes in cells and even transgenic animals expressing mutant regulatory proteins, it will be possible to determine whether and how a particular molecule operates upon the activation of Aurora kinase signaling cascade. Molecular engineering of fluorescent reporters on a desired molecule has emerged as an exciting way to correlate the cytological changes to molecular function of individual regulatory proteins in real time imaging of live cells. Such studies will consolidate protein-protein interactions that have been inferred from indirect techniques or in vitro test tube biochemistry into a physiological model for Aurora-mediated regulatory networking, and relate these data to molecular medicine of aberrant mitosis associated cell growth disorders such as cancers. A better understanding of the links that relate dynamics of mitotic apparatus to segregation of genomes will not only illustrate the precise function of Aurora kinases, but also provide a rationale for developing therapeutic targets of new anti-cancer drugs.

\section{ACKNOWLEDGEMENTS}

We also thank members of our group for insightful discussion during the preparation of this article. This work was supported by grants from Chinese 
Outstanding Young Scientist Award (39925018), Chinese Academy of Science (KSCX2-2-01), and American Cancer Society (RPG59282) to XY. XY is an Industrial Research Scholar of American Digestive Health Foundation, and Cheung Kong Scholar of Li Ka Shing Foundation.

\section{REFERENCES}

1. Nigg EA. Mitotic kinases as regulators of cell division and its checkpoints. Nat Rev Mol Cell Biol 2001; 2:21-32.

2. Zhang J, Fu CH, Miao Y et al. Protein kinase TTK interacts and colocalizes with CENP-E to the kinetochore of human cells. Chinese Science Bulletin 2002; 47:2005-9.

3. Chan CS, Botstein D. Isolation and characterization of chromosome-gain and increase-in-ploidy mutants in yeast. Genetics 1993; 135:677-91.

4. Glover DM, Leibowitz MH, Mlean DA et al. Mutations in Aurora prevent centrosome separation leading to the formation of monopolar spindles. Cell 1995; 81:95-105.

5. Schumacher JM, Ashcroft N, Donovan PJ et al. A highly conserved centrosomal kinase, AIR-1, is required for accurate cell cycle progression and segregation of developmental factors in Caenorhabditis elegans embryos. Development 1998; 125:4391-402.

6. Schumacher JM, Golden A, Donovan PJ. AIR-2: An Aurora/Ipl1-related protein kinase associated with chromosomes and midbody microtubules is required for polar body extrusion and cytokinesis in Caenorhabditis elegans embryos. J Cell Biol 1998; 143:1635-46.

7. Bischoff JR, Plowman GD. The Aurora/Ipl1p kinase family: regulators of chromosome segregation and cytokinesis. Trends in Cell Biology 1999; 9:454-9.

8. Giet R, Prigent C. Aurora/Ipl1p-related kinases, a new oncogenic family of mitotic serine-threonine kinases. J Cell Sci 1999; 112:3591-601.

9. Kimura M, Kotani S, Hattori T et al. Cell cycle-dependent expression and spindle pole localization of a novel human protein kinase, AIK, related to Aurora of Drosophila and yeast Ipl1. J Biol Chem 1997; 272:13766-71.

10. Yang SC, Huang $\mathrm{CH}$, Chen NJ, et al. Functional implication of human serine/threonine kinase, hAIK, in cell cycle progression. J Biomed Sci 2000; 7:484-93.

11. Hannak E, Kirkham M, Hyman AA et al. Aurora-A kinase is required for centrosome maturation in Caenorhabditis elegans. J Cell Biol 2001; 155:1109-15.

12. Roghi C, Giet R, Uzbekov R et al. The Xenopus protein kinase pEg2 associates with the centrosome in a cell cycledependent manner, binds to the spindle microtubules and is involved in bipolar mitotic spindle assembly. J Cell Sci 1998; 111:557-72.

13. Francisco L, Wang W, Chan CS. Type 1 protein phosphatase acts in opposition to Ipl1 protein kinase in regulating yeast chromosome segregation. Mol Cell Biol 1994; 14: 4731-40.

14. Francisco L, Chan CS. Regulation of yeast chromosome segregation by Ipl1 protein kinase and type 1 protein phosphatase. Cell Mol Biol Res 1994; 40:207-13.

15. Tung HY, Wang W, Chan CS. Regulation of chromosome segregation by Glc8p, a structural homolog of mammalian inhibitor 2 that functions as both an activator and an inhibitor of yeast protein phosphatase 1. Mol Cell Biol 1995; 15:6064-74.

16. Katayama H, Zhou H, Li Q, et al. Interaction and feedback regulation between STK15/BTAK/Aurora-A kinase and protein phosphatase 1 through mitotic cell division cycle. J Biol Chem 2001; 273:46219-24.

17. Littlepage LE, Wu H, Andresson T et al. Identification of phosphorylated residues that affect the activity of the mitotic kinase Aurora-A. Proc Natl Acad Sci U S A 2002; 99:15440-5.

18. Walter AO, Seghezzi W, Korver W, et al. The mitotic serine/ threonine kinase Aurora2/AIK is regulated by phosphorylation and degradation. Oncogene 2000; 19:4906-16.

19. Honda K, Mihara H, Kato Y et al. Degradation of human Aurora 2 protein kinase by the anaphase-promoting complex-ubiquitin-proteasome pathway. Oncogene 2000; 19:2812-9.

20. Kramer ER, Scheuringer N, Podtelejnikov AV et al. Mitotic regulation of the APC activator protein CDC20 and CDH1. Mol Biol Cell 2000; 11:1555-69.

21. Castro A, Arlot-Bonnemains Y, Vigneron S et al. APC/ Fizzy-Related targets Aurora-A kinase for proteolysis. EMBO Rep 2002; 3:457-62.

22. Taguchi S, Honda K, Sugiura K et al. Degradation of human Aurora-A protein kinase is mediated by hCdh1. FEBS Lett 2002; 519:59-65.

23. Castro A, Vigneron S, Bernis C et al. The D-Box-activating domain (DAD) is a new proteolysis signal that stimulates the silent D-Box sequence of Aurora-A. EMBO Rep 2002; 3:1209-14.

24. Kiat LS, Hui KM, Gopalan G. Aurora-A kinase interacting protein (AIP), a novel negative regulator of human Aurora-A kinase. J Biol Chem 2002; 277:45558-65.

25. Giet R, Uzbekov R, Cubizolles F et al. The Xenopus laevis Aurora-related protein kinase pEg2 associates with and phosphorylates the kinesin-related protein XlEg5. J Biol Chem 1999; 274:15005-13.

26. Mendez R, Murthy KG, Ryan K et al. Phosphorylation of CPEB by Eg2 mediates the recruitment of CPSF into an active cytoplasmic polyadenylation complex. Mol Cell $2000 ; 6: 1253-9$.

27. Mendez R, Hake LE, Andresson T et al. Phosphorylation of CPE binding factor by Eg2 regulates translation of cmos mRNA. Nature 2000; 404:302-7.

28. Berdnik D, Knoblich JA. Drosophila Aurora-A Is Required for Centrosome Maturation and Actin-Dependent Asymmetric Protein Localization during Mitosis. Curr Biol 2002; 12:640-7.

29. Giet R, McLean D, Descamps S et al. Drosophila Aurora A kinase is required to localize D-TACC to centrosomes and to regulate astral microtubules. J Cell Biol 2002; 156:437-51.

30. Kufer TA, Sillje HH, Korner R. Human TPX2 is required for targeting Aurora-A kinase to the spindle. J Cell Biol 
2002; 158:617-23.

31. Gruss OJ, Wittmann M, Yokoyama H et al. 2002. Chromosome-induced microtubule assembly mediated by TPX2 is required for spindle formation in HeLa cells. Nat Cell Biol 2002; 4:871-9.

32. Sakai H, Urano T, Ookata K et al. MBD3 and HDAC1, Two Components of the NuRD Complex, Are Localized at Aurora-A-positive Centrosomes in M Phase. J Biol Chem 2002; 277:48714-23.

33. Chen SS, Chang PC, Cheung YW et al. Suppression of the STK15 oncogenic activity requires a transactivation-independent p53 function. EMBO J 2002; 21:4491-9.

34. Adams RR, Carmena M, Earnshaw WC. Chromosomal passengers and the (Aurora) ABCs of mitosis. Trends in Cell Biology 2001; 11:49-54.

35. Uren AG, Wong L, Pakusch M et al. Survivin and the inner centromere protein INCENP show similar cell-cycle localization and gene knockout phenotype. Curr Biol 2000; 10:1319-28.

36. Severson AF, Hamill DR, Carter JC et al. The Aurorarelated kinase AIR-2 recruits ZEN-4/CeMKLP1 to the mitotic spindle at metaphase and is required for cytokinesis. Curr Biol 2000; 10:1162-71.

37. Bishop JD, Schumacher JM. Phosphorylation of the carboxyl terminus of inner centromere protein (INCENP) by the Aurora B Kinase stimulates Aurora B kinase activity. J Biol Chem 2002; 277:27577-80.

38. Bolton MA, Lan W, Powers SE et al. Aurora B kinase exists in a complex with survivin and INCENP and its kinase activity is stimulated by survivin binding and phosphorylation. Mol Biol Cell 2002; 13:3064-77.

39. Chen J, Jin S, Tahir SK et al. Survivin enhances Aurora-B kinase activity and localizes Aurora-B in human cells. J Biol Chem 2003; 278:486-90.

40. Gigoux V, L扝oste S, Raynaud F. Identification of Aurora kinases as RasGAP Src homology 3 domain-binding proteins. J Biol Chem 2002; 277:23742-6.

41. Kim JH, Kang JS, Chan CS. Sli15 associates with the Ipl1 protein kinase to promote proper chromosome segregation in Saccharomyces cerevisiae. J Cell Biol 1999; 145: 1381-94.

42. Kang J, Cheeseman IM, Kallstrom G et al. Functional cooperation of Dam1, Ipl1, and the inner centromere protein (INCENP)-related protein Sli15 during chromosome segregation. J Cell Biol 2001; 155:763-74.

43. Cheeseman IM, Anderson S, Jwa M et al. Phospho-regulation of kinetochore-microtubule attachments by the Aurora kinase Ipl1p. Cell 2002; 111:163-72.

44. Tanaka TU, Rachidi N, Janke C et al. Evidence that the Ipl1-Sli15 (Aurora kinase-INCENP) complex promotes chromosome bi-orientation by altering kinetochorespindle pole connections. Cell 2002; 108:317-29.

45. Wei Y, Yu L, Bowen J et al. Phosphorylation of histone H3 is required for proper chromosome condensation and segregation. Cell 1999; 97:99-109.

46. Hsu JY, Sun ZW, Li X, et al. Mitotic phosphorylation of histone H3 is governed by Ipl1/Aurora kinase and Glc7/ PP1 phosphatase in budding yeast and nematodes. Cell
$2000 ; 102: 1-20$.

47. Murnion ME, Adams RR, Callister DM et al. Chromatinassociated protein phosphatase 1 regulates Aurora-B and histone H3 phosphorytation. J Biol Chem 2001; 276: 26656-65.

48. Crosio C, Fimia GM, Loury R et al. Mitotic phosphorylation of histone H3: spatio-temporal regulation by mammalian Aurora kinases. Mol Cell Biol 2002; 22:874-85.

49. Goto H, Yasui Y, Nigg EA et al. Aurora-B phosphorylates Histone $\mathrm{H} 3$ at serine28 with regard to the mitotic chromosome condensation. Genes Cells 2002; 7:11-7.

50. Zeitlin SG, Barber CM. Differential regulation of CENPA and histone H3 phosphorylation in G2/M. J Cell Sci 2002; 114:653-61.

51. Zeitlin SG, Shelby RD, Sulivan KF. CENP-A is phosphorylated by Aurora B kinase and plays an unexpected role in completion of cytokinesis. J Cell Biol 2001; 155:114757.

52. Lange BM, Rebollo E, Herold A et al. Cdc37 is essential for chromosome segregation and cytokinesis in higher eukaryotes. EMBO J 2002; 21:5364-74.

53. Goto H, Yasui Y, Kawajiri A et al. Aurora-B regulates the cleavage furrow-specific vimentin phosphorylation in the cytokinetic process. J Biol Chem (in press)

54. Kallio MJ, McCleland ML, Stukenberg PT et al. Inhibition of Aurora B kinase blocks chromosome segregation, overrides the spindle checkpoint, and perturbs microtubule dynamics in mitosis. Curr Biol 2002; 12:900-5.

55. Murata-Hori M, Wang Y. The Kinase activity of Aurora B is required for kinetochore-microtubule interactions during mitosis. Curr Biol 2002; 12:894-9.

56. Liao H, Li G, Yen TJ. Mitotic regulation of microtubule cross-linking activity of CENP-E kinetochore protein. Science 1994; 265(5170):394-8.

57. Yao X, Abrieu A, Zheng Y et al. CENP-E forms a link between attachment of spindle microtubules to kinetochores and the mitotic checkpoint. Nat Cell Biol 2000; 2:484-91.

58. Yao X, Anderson KL, Cleveland DW. The microtubuledependent motor centromere-associated protein $\mathrm{E}$ (CENP-E) is an integral component of kinetochore corona fibers that link centromeres to spindle microtubules. J Cell Biol 1997; 139:435-47.

59. Kimura M, Matsuda Y, Yoshioka T. Cell cycle-dependent expression and centrosome localization of a third human Aurora/Ipl1-related protein kinase, AIK3. J Biol Chem 1999; 274:7334-40.

60. Ried T, Heselmeyer-Haddad K, Blegen H et al. Genomic changes defining the genesis, progression, and malignancy potential in solid human tumors: a phenotype/ genotype correlation. Genes Chromosomes Cancer 1999; 25:195-204.

61. Xie W, Li L, Cohen SN. Cell cycle-dependent subcellular localization of the TSG101 protein and mitotic and nuclear abnormalities associated with TSG101 deficiency. Proc Natl Acad Sci USA. 1998; 95:1595-600.

62. Brinkley BR. Managing the centrosome numbers game: from chaos to stability in cancer cell division. Trends in 
Cell Biology 2001; 11:18-21.

63. Lengauer C, Kinzler KW, Vogelstein B. Genetic instabilities in human cancers. Nature 1998; 396:643-9.

64. Sen S, Zhou H, White R. A. A putative serine/threonine kinase encoding gene BTAK on chromosome 20q13 is amplified and overexpressed in human breast cancer cell lines. Oncogene 1997; 14:2195-200.

65. Bischoff JR, Anderson L, Zhu Y et al. A homologue of Drosophila Aurora kinase is oncogenic and amplified in human colorectal cancers. EMBO J 1998; 17:3052-65.

66. Zhou H, Kuang J, Zhong L et al. Tumour amplified kinase STK15/BTAK induces centrosome amplification, aneuploidy and transformation. Nat Genet 1998; 20:189-93.

67. Tatsuka M, Katayama H, Ota T et al., Multinuclearity and increased ploidy caused by overexpression of the Aurora- and Ipl1-like midbody-associated protein mitotic kinase in human cancer cells. Cancer Res 1998; 58: 4811-6.

68. Tanaka T, Kimura M, Matsunaga K et al. Centrosomal kinase AIK1 is overexpressed in invasive ductal carcinoma of the breast. Cancer Res 1999; 59:2041-4.

69. Takahashi T, Futamura M, Yoshimi $\mathrm{N}$ et al. Centrosomal kinases, HsAIRK1 and HsAIRK3, are overexpressed in primary colorectal cancers. Jpn J Cancer Res 2000; 91: 1007-14.
70. Sakakura C, Hagiwara A, Yasuoka R et al. Tumour-amplified kinase BTAK is amplified and overexpressed in gastric cancers with possible involvement in aneuploid formation. Br J Cancer 2001; 84:824-31.

71. Miyoshi Y, Iwao K, Egawa C et al. Association of centrosomal kinase STK15/BTAK mRNA expression with chromosomal instability in human breast cancers. Int $J$ Cancer 2001; 92:370-3.

72. Meraldi P, Honda R, Nigg EA. Aurora-A overexpression reveals tetraploidization as a major route to centrosome amplification in p53-/- cells. EMBO J 2002; 21:483-92.

73. Borel F, Lohez OD, Lacroix FB. Multiple centrosomes arise from tetraploidy checkpoint failure and mitotic centrosome clusters in p53 and RB pocket protein-compromised cells. Proc Natl Acad Sci USA 2002; 99:981924.

74. Adams R. R. Eckley DM, Vagnarelli P et al. Human INCENP colocalizes with the Aurora-B/AIRK2 kinase on chromosomes and is overexpressed in tumour cells. Chromosoma 2001; 110:65-74. 